\title{
Quão Imperfeita é a Competição na Indústria Brasileira? Estimativas de Mark $U p$ Setorial entre 1996 e 2007
}

\author{
Claudio Lucinda \\ Professor - Universidade de São Paulo - Faculdade de Economia, Administração e \\ Contabilidade de Ribeirão Preto (FEA-RP/USP) \\ Endereço para contato: Av. Bandeirantes, 3900 - Monte Alegre - Ribeirão Preto \\ São Paulo - Brasil - CEP: 14040-900 - E-mail: claudiolucinda@usp.br
}

\section{Leandro Meyer}

Doutorando em Economia - Universidade de São Paulo, Escola Superior de Agricultura "Luiz de Queiroz" (ESALQ) - Endereço para contato: Av. Pádua Dias, 11 - Piracicaba - SP - Brasil CEP: 13418-900 - E-mail: leandro.meyer@gmail.com

Recebido em 14 de agosto de 2012. Aceito em 14 de agosto de 2013.

\section{Resumo}

O uso de medidas de produtividade na avaliação de políticas públicas baseadas na suposição de concorrência perfeita pode levar a conclusões incorretas sobre a sua eficácia. Nesse sentido, o presente estudo busca analisar quão distante da competição perfeita a indústria de transformação brasileira se encontra. Os resultados apontam que, em média, o preço é 2,41 vezes o custo marginal, rejeitando a validade da hipótese de concorrência perfeita e indicando que análises que não levam em conta tal padrão de competição geram conclusões errôneas. Os setores florestal e metalúrgico apresentaram os maiores mark ups (3,81 e 2,95 respectivamente), sendo tal conclusão robusta a retornos de escala e formas alternativas de mensuração.

\section{Palavras-Chave}

mark up, função de produção, setor industrial

\begin{abstract}
The use of productivity measures based on the assumption of perfect competition to evaluate public policies can lead to incorrect conclusions about them. In this sense, the present research aims to analyze how far from perfect competition the Brazilian industry is. The results indicated that the prices are, in general, 2.41 times marginal cost, which shows that the perfect competition hypothesis is not valid for the Brazilian industry and indicate that not considering the competition pattern lead to incorrect conclusions. The forest and metallurgy sectors had the highest mark ups (3.81 and 2.95 respectively), and these results are robust to assumptions about scale economies and different measures of mark up.
\end{abstract}

\section{Keywords}

mark up, production function, industrial sector

\section{JEL Classification}

D22, D43, L13 


\section{Introdução}

Desde estudos macroeconômicos que se baseiam em estimativas de Produtividade Total dos Fatores até estudos baseados em modelos de Equilíbrio Geral Computável, a ampla maioria dos estudos que buscam avaliar os efeitos de políticas setoriais se baseia na suposição de preço sendo igual a custo marginal. Esta premissa, que simplifica bastante tanto manipulações algébricas quanto cálculos numéricos, não é inócua. Desde o artigo de Hall (1986), estudos apontam que essa premissa de competição perfeita pode levar a conclusões incorretas. Mesmo para o caso brasileiro, já existem algumas evidências que apontam nesta direção, como os estudos de Clezar et al. (2010) e Ferreira e Guillén (2004), que estimam diferenças significativas entre preço e custo marginal para grande parte da indústria nacional.

Um exemplo de estudo que se baseia neste tipo de premissa é o de Domingues et al. (2012), em que os autores aplicam um modelo de equilíbrio geral ao setor industrial, obtendo estimativas do impacto da desoneração sobre a folha de salários na economia. Os resultados indicam que o impacto da desoneração fiscal é positivo. Contudo, a magnitude e até mesmo o sinal das variações simuladas devem sofrer alterações caso a hipótese de concorrência perfeita não seja válida empiricamente.

Outro exemplo, utilizando medidas agregadas de produtividade supondo concorrência perfeita, encontra-se no debate sobre a abertura comercial da economia brasileira e seus efeitos sobre a produtividade. ${ }^{l}$ Ferreira e Guillén (2004) encontram evidências de que o aumento da produtividade usualmente é superestimado ao empregarem uma metodologia que permite separar o efeito da diferença entre preço e custo marginal. Ademais, as estimativas mostram que os indicadores de competição, fator que usualmente é considerado um dos mais relevantes promotores da produtividade, não tiveram alterações significativas. Ou seja, diversos estudos empíricos superestimam o efeito da abertura comercial e da concorrência sobre a produtividade da indústria brasileira por ignorar a diferença entre preço e custo marginal, o que ressalta a importância de se estimar a relação entre estas variáveis.

1 Como é possível observar em Moreira (1999), Ferreira e Junior (2001), Garcia (2003) e Muendler (2004), por exemplo. 
Além de ser uma hipótese relevante para estudos aplicados, a relação entre preço e custo marginal também oferece informações importantes para recomendações de políticas públicas. A decisão do governo em estimular o fortalecimento de indústrias nacionais em determinados setores tem como contrapartida prejuízos para o consumidor brasileiro, já que confere poder de mercado aos grandes players nacionais. A esse respeito, índices agregados de mark up revelam desvios do ideal de concorrência perfeita que podem estar associados ao exercício de poder de mercado, o que deve ser evitado, ou pelo menos minimizado, por políticas de defesa da concorrência.

Nesse sentido, o presente trabalho possui como objetivo estimar a relação entre preço e custo marginal para uma amostra grande de indústrias brasileiras, usando dados da PIA-Empresa/IBGE. Para isto, serão utilizadas duas metodologias, a primeira delas a de Hall (1986), diretamente baseada no conceito de Resíduo de Solow da função de produção, de maneira semelhante à utilizada em Ferreira e Guillén (2004) e em Clezar et al. (2010).

Outro avanço importante do presente artigo, ainda sobre a relação entre preço e custo marginal, é que se adota aqui uma metodologia robusta a retornos crescentes ou decrescentes de escala. Loecker e Warzynski (2009) propõem uma forma de estimar a relação entre preço e custo marginal que parte diretamente das estimativas da função de produção, e as conclusões não dependem de premissas sobre retornos de escala. ${ }^{2}$ Dessa forma, a análise do resíduo de Solow e da função de produção apresentam características complementares e devem gerar constatações relevantes sobre a relação entre preço e custo marginal. Para obtenção de inferências sobre a relação entre preço e custo marginal da indústria brasileira, é preciso abordar os principais aspectos das metodologias a serem aplicadas. Para isso, os procedimentos de estimação do mark up por meio do resíduo de Solow e da função de produção são abordados a seguir. A análise será realizada em nível setorial. Por isso, as indústrias - correspondentes às classificações três dígitos na Classificação Nacional de Atividades Econômicas (CNAE) - serão agrupadas em setores na seção 3, na qual também é apresentada a base de dados. Na sequência, os resultados são analisados e, por fim, a conclusão encerra a pesquisa.

2 Até onde se conhece, o presente artigo é o primeiro a aplicar esta metodologia de Loecker e Warzynski (2009) para a indústria brasileira. 


\section{Metodologia}

O fato de o custo marginal não ser observável dificulta o estudo sobre o mark up. Metodologias que transcendem a simples análise de preços, custos observáveis e índices técnicos de produção surgiram e se difundiram durante os anos 1980, um período de importantes avanços em estudos empíricos de organização industrial. O ferramental analítico proposto por Hall (1986) é uma das formas mais relevantes de estimar os mark ups que surgiram neste contexto.

Ao analisar dados do uso de insumos e da produção das indústrias norte-americanas, Hall (1986) constatou que entre 1953 e 1984 o mark up foi superior à unidade, evidenciando que a hipótese de igualdade entre preço e custo marginal não era empiricamente válida para a maior parte das indústrias americanas naquele período. Uma das conclusões do estudo foi que, em fases de alto crescimento, as firmas produzem mais, vendendo por um preço que excede o custo marginal dos insumos. Os trabalhos de Domowitz et al. (1986) e de Shapiro (1987) foram os mais relevantes que seguiram esta linha metodológica. Ambos analisaram a indústria norte-americana partindo de tal abordagem e realizaram estimativas complementares que os levaram a constatações semelhantes.

Essa abordagem de estimação do mark up, que também será aplicada na presente pesquisa, parte da análise da função de produção $\mathrm{Q}=F(K ; N ; \theta)$, sendo $Q$ o valor agregado, $K$ o capital, $N$ o trabalho e $\theta$ a mudança técnica. Algumas hipóteses devem ser assumidas e manipulações algébricas devem ser empregadas para que a função de produção revele constatações relevantes. É considerado o caso em que a mudança técnica não altera as taxas marginais de substituição dos fatores de produção, simplesmente aumentando ou diminuindo o produto obtido dado o uso dos insumos. Como demonstrado por Hall (1988, p. 926), tais hipóteses levam à seguinte especificação:

$$
\Delta q_{i t}=\mu_{i} \alpha_{i}^{n} \Delta n_{i t}+\theta_{i}+e_{t}
$$

$\mathrm{Na}$ equação acima, a mudança técnica é dada por uma taxa de crescimento $\theta_{i}$ acrescida de um termo que representa o choque de produtividade, definido como $e_{t}$. As variáveis em minúsculo são definidas como a variação do valor agregado $\left(\Delta q_{i t}\right)$ e do trabalho $\left(\Delta n_{i t}\right)$, ambas descontadas da variação do capital. A contribuição do trabalho no 
produto é definida como $\alpha_{i t}^{n}=w_{i t} N_{i t} / p_{i t} Q_{i t}$, sendo $w_{i t}$ o salário e $p_{i t}$ o preço do produto, e o mark up é considerado constante no período de análise, de forma que $\mu_{i=} \rho_{i} / x_{i}$, em que $p_{i}$ é o preço e $x_{i}$ é o custo marginal. A Equação (1) expressa a ideia básica da metodologia de estimação do mark up. Na regressão de $\Delta q_{i t}$ contra $\Delta n_{i t}$, o coeficiente obtido é igual à contribuição do trabalho no valor agregado multiplicada pelo mark up ( $\mu_{i} \alpha_{i}^{n}$ ). As estimativas de mark up são obtidas por meio da divisão do coeficiente obtido em (1) pela contribuição do trabalho no valor agregado $\left(\alpha_{i}^{n}\right)$, que pode ser obtida diretamente dos dados.

É possível entender a Equação (1) da seguinte forma: em momentos de expansão do valor do produto, é possível que esta não seja justificada apenas pela expansão no uso de insumos e em seu valor, havendo espaço também para o aumento do preço em relação ao custo marginal. Supõe-se aqui que seja possível isolar este efeito ao dividir o coeficiente da regressão pela contribuição do trabalho no produto. Como ressaltado pelo seu próprio autor, neste ponto fica evidente a principal limitação da metodologia: retornos não constantes de escala também podem explicar expansão do produto não relacionada ao uso de insumos, implicando que as estimativas de mark up são viesadas em tais situações.

Esse procedimento para estimação do mark up requer o uso de variáveis instrumentais. Por isso é necessária a escolha de um instrumento que deve causar importantes variações no produto e no emprego e não ter correlação com os choques de produtividade. Hall (1988) utilizou gastos militares, preço mundial do petróleo e partido do presidente norte-americano, variáveis que não parecem ser adequadas para o caso da indústria brasileira. Outro trabalho relevante que faz uso das variáveis instrumentais é Charles e Perloff (1993), com destaque para o uso da oferta de moeda. Sobre a literatura nacional, o único trabalho encontrado que aborda a escolha de variáveis instrumentais para a aplicação desta metodologia é Ferreira e Guillén (2004). Apesar de os autores não utilizarem variáveis instrumentais em suas estimações por afirmarem não haver mudanças significativas nos resultados com o emprego destas, é possível encontrar no trabalho indicações sobre os instrumentos a serem utilizados, com destaque para taxa de câmbio. ${ }^{3}$

3 Além de taxa de câmbio e oferta monetária, outras variáveis foram testadas como instrumentos, como horas trabalhadas, população economicamente ativa e crédito destinado à 
Outra opção metodológica passa pela estimação direta da função de produção. De acordo com Mundlak (1963), as estimativas das elasticidades dos fatores de produção podem gerar evidências sobre concorrência. Tais evidências podem ser obtidas por meio da comparação entre as elasticidades dos fatores de produção e a contribuição dos insumos, como no caso da metodologia exposta na seção anterior. Caso a hipótese de concorrência perfeita seja válida, estes dois valores devem ser estatisticamente iguais. Baseando-se nesta comparação, Loecker e Warzynski (2009) e Loecker (2011) propõem uma forma de mensurar o mark up por meio da divisão entre a elasticidade do trabalho pela contribuição do mesmo insumo no valor agregado da seguinte forma:

$$
\mu_{i}=\frac{\beta_{i}^{n}}{\alpha_{i}^{n}}
$$

sendo $\mu_{i}$ o mark up, $\beta_{i}^{n}$ a elasticidade-valor agregado do trabalho e $\alpha_{i}^{n}$ a contribuição do trabalho no valor agregado. Nota-se aqui que há grande semelhança entre esse procedimento e o que parte da análise do resíduo de Solow. Novamente, é considerada a influência do trabalho no valor agregado, anteriormente obtida por meio da regressão entre as variáveis e neste caso obtida por meio da estimação dos parâmetros da função de produção. Evidentemente, é necessário obter estimativas não viesadas de tais parâmetros.

Os procedimentos de estimação da função de produção constituem uma área que possui literatura bastante desenvolvida. O primeiro aspecto relevante é a forma funcional considerada. No presente estudo é utilizada a função de produção Cobb-Douglas por esta ser amplamente difundida na literatura. Outro aspecto que se destaca sobre a estimação da função de produção é que a estimativa via mínimos quadrados ordinários (MQO) é viesada. Griliches e Mairesse (1995) afirmam que cada abordagem de tratamento desse viés corresponde a uma interpretação diferente sobre a fonte da endogeneidade, ou seja, há diversas abordagens possíveis para obtenção de tais estimativas.

Na presente pesquisa, é aplicada a metodologia que parte da especificação dinâmica da função de produção Cobb-Douglas. A forma funcional da função Cobb-Douglas, expandida para acomodar efeitos

pessoa física. Porém o uso de tais variáveis gerou resultados não factíveis (como mark ups negativos, por exemplo). 
fixos de ano e de tempo juntamente com o termo erro econométrico, é dada pela seguinte equação:

$$
q_{i t}=\beta^{k} k_{i t}+\beta^{n} n_{i t}+\gamma_{t}+\eta_{i}+m_{i t}
$$

Em que $q_{i t}$ é o $\log$ do valor agregado, $k_{i t}$ é o $\log$ do capital, $n_{i t}$ é o $\log$ do trabalho, $\beta^{k}$ é a elasticidade-valor agregado do capital, $\beta^{n}$ é a elasticidade-valor agregado do trabalho, $\left(\eta_{i}\right)$ é um componente de efeitos não observados específico de cada indústria, $\left(\gamma_{t}\right)$ é um intercepto específico de ano e $\left(m_{i t}\right)$ são os erros de mensuração não serialmente correlacionados. A especificação dinâmica é consequência da suposição de que os choques de produtividade $\left(v_{i t}\right)$ apresentam persistência, de forma que $v_{i t}=\rho v_{i, t-1}+e_{i t}$, sendo $e_{i t} \sim M A(0)$ . Considerando este comportamento, Blundell e Bond (1998, p.3) chegam à seguinte especificação, obtida a partir da diferença entre a equação acima e sua versão defasada após um período:

$$
\begin{gathered}
q_{i t}=\beta^{k} k_{i t}-\rho \beta^{k} k_{i t-1}+\beta^{n} n_{i t}-\rho \beta^{n} n_{i t-1}+\rho q_{i t-1}+\left(\gamma_{t}-\rho \gamma_{t-1}\right) \\
+\left[\eta_{i}(1-\rho)+e_{i t}+m_{i t}-\rho m_{i t-1}\right]
\end{gathered}
$$

$\mathrm{ou}$

$$
q_{i t}=\pi_{1} k_{i t}-\pi_{2} k_{i t-1}+\pi_{3} n_{i t}-\pi_{4} n_{i t-1}+\pi_{5} q_{i t-1}+\gamma_{t}^{*}+\left(\eta_{i}^{*}+w_{i t}^{*}\right)
$$

Os termos modificados na Equação (5) são $\gamma_{t}^{*}=\gamma_{t}-\rho \gamma_{t-1}, \eta_{i}^{*}=$ $\eta_{i}(1-\rho)$ e $w_{i t}^{*}=e_{i t}+m_{i t}-\rho m_{i t-1}$. A passagem de (4) para (5) está sujeita a duas restrições não lineares: $\pi_{2}=-\pi_{1} . \pi_{5}$ e $\pi_{4}=-\pi_{3} \pi_{5}$. Com estimativas consistentes do vetor de parâmetros $\pi=\left(\pi_{1}, \pi_{2}\right.$, $\left.\pi_{3}, \pi_{4}, \pi_{5}\right)$ e $\operatorname{var}(\pi)$, estas restrições podem ser impostas e testadas para obtenção dos parâmetros do vetor de interesse $\left(\beta^{n}, \beta^{n}, \rho\right)$.

As restrições devem ser validadas pelo teste dos fatores comuns (COMFAC), que possui como hipótese nula que tais restrições estão sendo satisfeitas (Blundell e Bond, 1998). Acerca do procedimento de estimação empregado, de acordo com Cameron e Trivedi (2005), uma importante constatação sobre a estrutura de painel dinâmico é que as estimativas dos parâmetros $\pi$ obtidas via MQO são inconsistentes. $\mathrm{O}$ caminho apontado pela literatura foi o de reti- 
rar a primeira diferença e instrumentalizar a equação com as variáveis em nível (Anderson e Hsiao (1981) e Arellano e Bond (1991)). Tal abordagem faz uso da premissa de que a quantidade de um fator de produção utilizado no ano anterior tem grande correlação com a quantidade utilizada no ano seguinte e não tem correlação com os choques de produtividade do ano seguinte. Apesar de corrigirem o problema, tais metodologias usualmente apresentam coeficientes do capital baixos e não significantes, além de retornos de escala pouco plausíveis, conforme exposto em Griliches e Mairesse (1995).

Para Blundell e Bond (1998), esse problema ocorre porque as séries utilizadas apresentam grande persistência, o que faz com que a variável em nível e a variável em diferença guardem pouca correlação. Para melhorar esse aspecto da instrumentalização, os autores assumem condições de momento adicionais, que podem ser testadas, e que permitem a estimação conjunta da equação em nível instrumentalizada pela defasagem das diferenças, gerando o estimador System GMM. Os autores mostram por meio de simulações que este procedimento aumenta o nível de significância dos coeficientes estimados e gera estimativas de retorno de escala mais plausíveis.

Esse procedimento aumenta o conjunto de informações utilizado na instrumentalização por supor condições de momento adicionais, sendo que sua adequabilidade depende da validade de tais condições. Para isso, o teste de Sargan e o teste de Sargan em diferenças (D-Sargan) devem ser realizados. O teste de Sargan possui como hipótese nula que as condições de momento assumidas para o estimador GMM são adequadas, enquanto que a hipótese nula do teste D-Sargan é que as condições adicionais de momento assumidas para o System GMM são adequadas. Dessa forma, tendo sido realizada a estimação dos parâmetros, deve-se observar o COMFAC, os testes Sargan e D-Sargan. A literatura também indica a necessidade da realização do teste de autocorrelação serial de segunda ordem do resíduo $A R(2)$, que possui como hipótese nula a não existência de autocorrelação deste termo. Esse teste é necessário para avaliar a validade das premissas do modelo com relação às defasagens utilizadas na instrumentalização. Diferente das estimativas de mark up obtidas por meio do resíduo de Solow, as obtidas por meio da função de produção não são afetadas pela natureza dos retornos de escala. Por isso, adicionalmente, será avaliada a presença de retornos constantes 
de escala para os setores, o que auxilia a comparação das estimativas de mark up obtidas das duas formas.

Há diferentes formas de se obter inferências a esse respeito. No presente estudo, lançamos mão de um teste calculado a partir da função de produção por este ser bastante semelhante ao procedimento aplicado para estimação da função de produção. A função de produção dinâmica pode ser rearranjada da seguinte forma:

$$
\begin{gathered}
q_{i t}-k_{i t}=\beta_{i}^{n}\left(n_{i t}-k_{i t}\right)-\rho \beta_{i}^{n}\left(n_{i t-1}-k_{i t-1}\right)+\left(\beta_{i}^{n}+\beta_{i}^{k}-1\right) k_{i t} \\
-\rho\left(\beta_{i}^{n}+\beta_{i}^{k}-1\right) k_{i t-1}+\rho\left(q_{i t-1}-k_{i t-1}\right)+\eta_{i}^{*}+e_{i t}
\end{gathered}
$$

ou

$q_{i t}-k_{i t}=\pi_{1}\left(n_{i t}-k_{i t}\right)+\pi_{2}\left(n_{i t-1}-k_{i t-1}\right)+\pi_{3} k_{i t}+\pi_{4} k_{i t-1}+\pi_{5}\left(q_{i t-1}-k_{i t-1}\right)+\eta_{i}^{*}+e_{i t}$

A passagem de (6) para (7) implica duas restrições não lineares: $\pi_{4}$ $=-\pi_{3} \pi_{5}$ e $\pi_{2}=-\pi_{1} . \pi_{5}$. De maneira análoga à estimação da função de produção, estas restrições podem ser testadas pelo COMFAC. Neste caso, ao testarmos a restrição $\pi_{4}=-\pi_{3} \pi_{5}$ está sendo indiretamente testada a hipótese de que os coeficientes dos insumos somam a unidade, como é possível observar ao comparar as Equações (6) e (7). Dessa forma, o teste dessa restrição pode ser interpretado como um teste cuja hipótese nula é de retornos constantes de escala, o que será utilizado para verificação desta hipótese.

\section{Dados}

Os dados utilizados no presente estudo provêm da Pesquisa Industrial Anual (PIA-Empresa). Foram consideradas as indústrias classificadas como de extração e de transformação, o que totalizou 104 indústrias. O período analisado vai de 1996 a $2007 .^{4}$

4 Para os anos mais recentes, foram realizadas alterações na classificação das indústrias, sendo 
A estimação da função de produção por meio do System GMM requer o agrupamento de indústrias em setores. A esse respeito, na Classificação Nacional de Atividades Econômicas (CNAE), os dois primeiros algarismos englobam indústrias com atividades semelhantes, o que serviu como critério principal para a definição dos setores a serem analisados. Este critério, no entanto, gerou alguns grupos com pequeno número de indústrias. Para esses casos, foi analisado se tais indústrias possuem atividades semelhantes a alguns dos setores já definidos pelo primeiro critério. As características levadas em conta são o tipo de atividade realizada e o uso dos bens produzidos. Como ainda assim restaram grupos com poucas observações, estes foram classificados em um grupo denominado "outros". Este grupo não gera resultados de interesse analítico por abrigar indústrias de atividades muito diversas. A adoção de tais critérios gerou onze setores além do grupo composto pelas indústrias classificadas em outros. O número de indústrias em cada setor varia entre seis e doze, como é possível observar na Tabela (1), que também descreve o código das indústrias que compõem cada setor.

Tabela 1 - Lista dos setores definidos

\begin{tabular}{lcc}
\hline \multicolumn{1}{c}{ Setor } & № Indústrias & \multicolumn{1}{c}{ Código das Indústrias $^{5}$} \\
\hline Extrativista & 6 & $10.0 ; 11.2 ; 13.1 ; 13.2 ; 14.1 ; 14.2$ \\
Alimentos & 9 & $15.1 ; 15.2 ; 15.3 ; 15.4 ; 15.5 ; 15.6 ; 15.7 ; 15.8 ; 15.9$ \\
Têxtil & 12 & $17.1 ; 17.2 ; 17.3 ; 17.4 ; 17.5 ; 17.6 ; 17.7 ; 18.1 ; 18.2 ; 19.1 ; 19.2 ; 19.3$ \\
Florestal & 6 & $20.1 ; 20.2 ; 21.1 ; 21.2 ; 21.3 ; 21.4$ \\
Química & 9 & $24.1 ; 24.2 ; 24.3 ; 24.4 ; 24.5 ; 24.6 ; 24.7 ; 24.8 ; 24.9$ \\
Minerais não metálicos & 5 & $26.1 ; 26.2 ; 26.3 ; 26.4 ; 26.9$ \\
Metalurgia básica & 10 & $27.1 ; 27.2 ; 27.3 ; 27.4 ; 27.5 ; 28.1 ; 28.2 ; 28.3 ; 28.4 ; 28.9$ \\
Máquinas e equipamentos & 8 & $29.1 ; 29.2 ; 29.3 ; 29.4 ; 29.5 ; 29.6 ; 29.7 ; 29.8$ \\
Eletroeletrônico & 12 & $30.1 ; 30.2 ; 31.1 ; 31.2 ; 31.3 ; 31.4 ; 31.5 ; 31.6 ; 31.9 ; 32.1 ; 32.2 ; 32.3$ \\
Equipamentos gerais & 5 & $33.1 ; 33.2 ; 33.3 ; 33.4 ; 33.5$ \\
Veículos automotores & 9 & $34.1 ; 34.2 ; 34.3 ; 34.4 ; 34.5 ; 35.1 ; 35.2 ; 35.3 ; 35.9$ \\
Outros & 13 & $16.0 ; 22.1 ; 22.2 ; 22.3 ; 23.1 ; 23.2 ; 23.4 ; 25.1 ; 25.2 ; 36.1 ; 36.9 ; 37.1 ; 37.2$ \\
\hline
\end{tabular}

Fonte: Elaborado pelos autores com dados da PIA.

Com as indústrias classificadas em setores, a etapa seguinte no que se refere à organização da base de dados é a descrição das variáveis

que a CNAE 2.0 passou a ser utilizada no lugar da 1.0. Com isso, algumas indústrias que existiam na amostra que abrange o período entre 1996 e 2007 deixaram de existir nas pesquisas seguintes.

5 A lista completa dos códigos e de suas indústrias correspondentes está disponível em http://www.cnae.ibge.gov.br/ 
utilizadas. Busca-se obter séries para valor agregado, trabalho e capital, além da contribuição do trabalho no produto $\left(\alpha_{i}^{n}\right)$.

Para o valor agregado, foi utilizado o valor da transformação industrial dividido pelo número de firmas de cada indústria. Para a construção da série trabalho foi utilizado o número médio de trabalhadores por firma, multiplicado pela jornada anual média de trabalho de 2.112 horas/homem. Para a série de capital, o presente trabalho faz uso do método do estoque perpétuo, que considera um estoque de capital para o primeiro ano da amostra, acrescentando investimentos e retirando uma taxa de depreciação fixa, gerando os valores da série para os anos restantes. Tais procedimentos seguem o proposto por Garcia (2003).

Para a construção do estoque de capital para o ano inicial foram calculados os coeficientes da distribuição funcional da renda. O coeficiente do trabalho $\left(\alpha_{i}^{n}\right)$ foi obtido dividindo a massa total de salários e remunerações, incluídos encargos sociais, pelo valor adicionado. O coeficiente do capital $\left(\alpha_{i}^{k}\right)$ é o complementar do coeficiente do trabalho. O estoque de capital é estimado a partir de valores conhecidos: o valor agregado, o custo total da mão de obra e o número médio de empregados por ano.

Sendo $k_{i t}$ o estoque de capital, $q_{i t}$ o valor agregado e $W_{i t}$ o valor médio aplicado em trabalho, o estoque de capital pode ser calculado como proposto por Garcia (2003):

$$
k_{i t}=\frac{\left(q_{i t}\right)^{\frac{1}{\alpha_{i}^{k}}}}{\left(W_{i}\right)^{\frac{1-\alpha_{i}^{k}}{\alpha_{i}^{k}}}}
$$

Dessa forma, tendo o estoque de capital de 1996 calculado, é descontada uma taxa de $5 \%$ referente à depreciação e somado o valor médio investido em aquisições e melhorias do ativo imobilizado por firma, séries disponíveis na PIA. Chega-se assim à série de capital utilizada. As médias entre os anos de 1996 e 2007 do valor agregado, do trabalho empregado, do capital e dos coeficientes do capital e do trabalho para cada setor podem ser observadas em anexo. 
Também em anexo está a evolução da média para a indústria de transformação de cada uma dessas variáveis. Por fim, a respeito das variáveis instrumentais a serem utilizadas na análise do resíduo de Solow, estas possuem as seguintes fontes: para a oferta de moeda, foi utilizada a série "Papel moeda em poder público"; e para a taxa de câmbio foi utilizada a "Taxa de câmbio comercial - compra - média", ambas disponibilizadas pelo Banco Central.

\section{Resultados}

O primeiro aspecto a ser avaliado sobre os resultados da análise do resíduo de Solow é a influência dos instrumentos sobre as estimativas. Foi possível constatar que a instrumentalização de fato afeta os resultados. ${ }^{6}$ Contudo, o uso conjunto das variáveis instrumentais selecionadas (taxa de câmbio e oferta monetária) gerou estimativas de mark up inferiores à unidade para mais da metade da amostra.

Como as estimativas realizadas representam o resultado da divisão do preço pelo custo marginal, valores inferiores à unidade indicam que o custo marginal excede o preço. Este tipo de resultado não é consistente com um equilíbrio de longo prazo na indústria, mesmo em competição perfeita, se considerarmos que nesta situação há prejuízo para as empresas, algo que dificilmente se manteria em indústrias e setores inteiros ao longo do período analisado. Dessa forma, tais estimativas sugerem que há problemas com o uso de taxa de câmbio e oferta monetária como variáveis instrumentais. O uso das variáveis instrumentais separadamente demonstrou que as estimativas obtidas somente por meio de taxa de câmbio como instrumentos não gerou este problema. Por isso, os resultados utilizados para a análise foram os obtidos por meio do emprego somente da taxa de câmbio como instrumento.

Para obtenção do mark up por meio da função de produção em nível setorial, foi realizada a estimação dos parâmetros da fun-

6 O resíduo de Solow foi regredido contra os instrumentos como forma de atestar a adequabilidade dos instrumentos, conforme proposto por Hall (1986). Os resultados mostraram relação positiva entre as variáveis, o que de acordo com o autor atesta a influência dos instrumentos, sendo que a estatística $F$ da regressão da taxa de câmbio foi de $(435,94)$ e a da regressão da oferta monetária foi de $(87,64)$. 
ção de produção por meio do System GMM para cada setor. ${ }^{7}$ Alguns setores apresentaram problemas na instrumentalização, o que tornou necessária a estimação com o uso de diferentes defasagens como instrumento. Na Tabela (3) são apresentadas as estimativas dos coeficientes da função de produção com instrumentalização a partir da segunda defasagem para a maior parte dos setores e com defasagens maiores para o setor químico, além dos setores de metalurgia básica, máquinas e equipamentos, eletroeletrônico, equipamentos gerais e veículos automotores. ${ }^{8} \mathrm{Na}$ mesma tabela também são apresentados os resultados dos testes realizados.

Tabela 2 - Estimativa dos parâmetros da função de produção via System GMM

\begin{tabular}{|c|c|c|c|c|c|c|}
\hline & Extrativista & Alimentos & Têxtil & Florestal & Química** & $\begin{array}{c}\text { Minerais não } \\
\text { metal. }\end{array}$ \\
\hline \multirow{2}{*}{$\beta_{i}^{n}$} & 0,925 & 0,604 & 1,137 & 1,338 & 0,787 & 0,868 \\
\hline & $(0,009)$ & $(0,083)$ & $(0,036)$ & $(0,042)$ & $(0,185)$ & $(0,019)$ \\
\hline \multirow{2}{*}{$\beta_{i}^{k}$} & 0,207 & 0,396 & 0,339 & 0,298 & 0,531 & 0,497 \\
\hline & $(0,077)$ & $(0,117)$ & $(0,051)$ & $(0,035)$ & $(0,155)$ & $(0,056)$ \\
\hline Comfac & 0,057 & 0,103 & 0,293 & 0,068 & 0,810 & 1,000 \\
\hline Sargan & 0,054 & 0,575 & 0,765 & 0,105 & 0,251 & 0,099 \\
\hline D-Sargan & 0,567 & 1,000 & 1,000 & 0,324 & 0,966 & 0,549 \\
\hline \multirow[t]{2}{*}{$\mathrm{AR}(2)$} & 0,217 & 0,883 & 0,671 & 0,830 & 0,173 & 0,437 \\
\hline & Metal. básica** & Máq. e equi.. ${ }^{\star \star \star}$ & Eletro eletrônico* & Equi. gerais ${ }^{\star \star *}$ & $\begin{array}{l}\text { Veículos } \\
\text { auto. }{ }^{* *}\end{array}$ & Outros \\
\hline \multirow{2}{*}{$\beta_{i}^{n}$} & 1,246 & 0,768 & 0,830 & 1,073 & 1,208 & 0,867 \\
\hline & $(0,166)$ & $(0,221)$ & $(0,039)$ & $(0,054)$ & $(0,066)$ & $(0,066)$ \\
\hline \multirow{2}{*}{$\beta_{i}^{k}$} & 0,219 & 0,290 & 0,571 & 0,405 & 0,339 & 0,378 \\
\hline & $(0,086)$ & $(0,032)$ & $(0,342)$ & $(0,135)$ & $(0,049)$ & $(0,284)$ \\
\hline Comfac & 0,811 & 0,590 & 0,784 & 1,000 & 0,976 & 0,306 \\
\hline Sargan & 0,063 & 0,961 & 0,992 & 0,976 & 0,698 & 0,000 \\
\hline D-Sargan & 0,244 & 0,590 & 1,000 & 0,583 & 0,389 & 0,000 \\
\hline $\operatorname{AR}(2)$ & 0,238 & 0,167 & 0,873 & 0,514 & 0,394 & 0,033 \\
\hline
\end{tabular}

P-valor dos testes apresentados. Comfac: Ho = Restrições dos fatores comuns são válidas; Sargan e Hansen: Ho = Instrumentalização para GMM adequada; D-Sargan e D-Hansen: Ho = Instrumentalização para o System GMM adequada; AR(2): Não há correlação serial de segunda ordem do resíduo. Desvio padrão entre parênteses. *A equação em diferença foi instrumentalizada a partir da quarta defasagem; ${ }^{* *}$ A equação em diferença foi instrumentalizada a partir da sétima defasagem; ${ }^{* * *}$ A equação em diferença foi instrumentalizada a partir da nona defasagem.

Fonte: Resultados da pesquisa.

7 Os resultados obtidos por Pooled OLS e por Difference GMM são apresentados em anexo.

8 Foram testadas diversas defasagens para instrumentalização da equação em diferença destes setores, sendo que as instrumentalizações apresentadas foram as que geraram os melhores resultados em termos dos testes econométricos e da significância dos coeficientes estimados. 
O mark up é obtido por meio da divisão da estimativa do coeficiente do trabalho calculado pela função de produção $\left(\beta_{i}^{n}\right)$ pela contribuição deste insumo no valor agregado $\left(\alpha_{i}^{n}\right)$ conforme visto na seção 2.2. Na Tabela (4) são expostos os mark ups obtidos por meio dessa divisão, bem como os obtidos por meio do resíduo de Solow, a soma dos coeficientes estimados pela função de produção e o p-valor do teste de retornos constantes de escala.

Tabela 3 - Retornos de escala, coeficientes da função de produção e estimativas de mark $u p$

\begin{tabular}{|c|c|c|c|c|}
\hline \multirow[b]{2}{*}{ Setores } & \multirow[b]{2}{*}{ Retornos constantes de escala * } & \multirow[b]{2}{*}{$\beta_{i}^{n}+\beta_{i}^{k}$} & \multicolumn{2}{|c|}{ Mark Up } \\
\hline & & & Resíduo de Solow & Função de produção \\
\hline \multirow{2}{*}{ Veículos automotores } & 0,000 & 1,546 & 2,392 & 2,513 \\
\hline & & $(0,103)$ & $(0,440)$ & $(0,112)$ \\
\hline \multirow{2}{*}{ Florestal } & 0,021 & 1,636 & 3,483 & 3,812 \\
\hline & & $(0,078)$ & $(0,641)$ & $(0,121)$ \\
\hline \multirow{2}{*}{ Extrativista } & 0,002 & 1,132 & 3,646 & 2,417 \\
\hline & & $(0,167)$ & $(0,671)$ & $(0,235)$ \\
\hline \multirow{2}{*}{ Têxtil } & 0,294 & 1,476 & 2,173 & 2,289 \\
\hline & & $(0,087)$ & $(0,400)$ & $(0,072)$ \\
\hline \multirow{2}{*}{ Metalurgia básica } & 0,074 & 1,465 & 2,855 & 2,955 \\
\hline & & $(0,252)$ & $(0,525)$ & $(0,394)$ \\
\hline \multirow{2}{*}{ Equipamentos gerais } & 0,085 & 1,478 & 2,507 & 2,435 \\
\hline & & $(0,174)$ & $(0,461)$ & $(0,089)$ \\
\hline \multirow{2}{*}{ Alimentos } & 0,524 & 1,001 & 3,909 & 2,022 \\
\hline & & $(0,200)$ & $(0,719)$ & $(0,277)$ \\
\hline \multirow{2}{*}{ Química } & 0,950 & 1,318 & 3,387 & 2,383 \\
\hline & & $(0,340)$ & $(0,623)$ & $(0,560)$ \\
\hline \multirow{2}{*}{$\begin{array}{l}\text { Minerais não } \\
\text { metálicos }\end{array}$} & 0,053 & 1,365 & 3,254 & 2,187 \\
\hline & & $(0,075)$ & $(0,598)$ & $(0,047)$ \\
\hline \multirow{2}{*}{$\begin{array}{l}\text { Máquinas e } \\
\text { equipamentos }\end{array}$} & 0,706 & 1,057 & 2,274 & 1,598 \\
\hline & & $(0,094)$ & $(0,418)$ & $(0,130)$ \\
\hline \multirow{2}{*}{ Eletroeletrônico } & 0,719 & 1,401 & 2,682 & 1,884 \\
\hline & & $(0,563)$ & $(0,493)$ & $(0,502)$ \\
\hline
\end{tabular}

*P-valor dos testes apresentados $(\mathrm{Ho}=$ retornos constantes de escala).

Desvio padrão entre parênteses.

Fonte: Resultados da pesquisa. 
Antes da análise dos resultados, é necessário abordar novamente a questão da agregação das indústrias em setores. Esta se fez necessária para que a estimação da função de produção por meio do System GMM fosse possível. Contudo, pode haver heterogeneidade dentro dos grupos de análise. Por isso, quanto mais semelhantes as características das indústrias forem no que se refere à produção e à preferência dos consumidores por seus produtos, menor deve ser a heterogeneidade dentro dos setores e mais próximos da realidade de cada indústria devem ser os mark ups setoriais estimados.

Como as estimativas obtidas por meio do Resíduo de Solow podem ser obtidas em nível industrial, o cálculo do mark up também foi realizado neste nível de agregação por meio desta metodologia, sendo que o desvio-padrão das estimativas das indústrias em relação à média setorial gera uma evidência acerca da heterogeneidade das indústrias componentes de cada setor. ${ }^{9}$ Este cálculo mostrou que a maior parte dos setores apresenta desvio inferior à unidade. Os setores de alimentos, florestal e de minerais não metálicos apresentaram desvio-padrão superior à unidade pelo fato de uma de suas indústrias apresentarem estimativas muito maiores que as demais (a saber, produção de óleos em alimentos, produção de celulose em florestal e produção de cimento em minerais não metálicos). Com exceção de tais indústrias, os resultados obtidos por meio do resíduo de Solow em nível industrial indicam pouca heterogeneidade dentro dos setores. Apenas no caso dos setores de metalurgia básica e extrativista as estimativas em nível industrial indicam que há maior heterogeneidade, com desvios superiores à unidade.

A respeito da relação entre as estimativas obtidas por meio das duas metodologias empregadas, a análise dos retornos de escala é a principal forma de comparar os resultados, uma vez que esta hipótese afeta os resultados obtidos por meio do resíduo de Solow, mas não afeta os mark ups estimados pela função de produção. A esse respeito, Charles e Perloff (1993) utilizam simulações para mostrar que os mark ups obtidos por meio do resíduo de Solow são subestimados sob retornos crescentes de escala e sobreestimados sob retornos decrescentes de escala.

9 As estimativas obtidas por meio do resíduo de Solow em nível industrial são apresentadas no anexo. 
Há diferença entre os coeficientes estimados somente para o setor de alimentos, considerando o nível de significância de 5\%. Esta igualdade entre as estimativas se deve essencialmente aos elevados desvios - padrão dos mark ups obtidos por meio do resíduo de Solow. No caso em que a hipótese de retornos constantes de escala não foi rejeitada, isto é, para todos os setores exceto de veículos automotores, florestal e extrativista, esta igualdade entre os resultados é esperada.

Para os casos dos setores veículos automotores e florestal, as somas dos coeficientes da função de produção indicam que há retornos crescentes de escala nestes setores, o que deve gerar um viés negativo nos mark ups calculados por meio do resíduo de Solow. Apesar de não haver diferença estatisticamente significativa entre os mark ups, as estimativas obtidas por meio do resíduo de Solow de fato são inferiores às obtidas por meio da função de produção para tais setores. Para o setor extrativista, a soma dos coeficientes é próxima da unidade, de forma que não é possível afirmar se há retornos crescentes ou decrescentes de escala. O mark up estimado por meio do resíduo de Solow é superior ao estimado por meio da função de produção, o que sugere a presença de retornos decrescentes de escala.

Dessa forma, nos casos em que a hipótese de retornos constantes de escala não foi rejeitada, apenas para o setor de alimentos os resultados não seguem o indicado por Charles e Perloff (1993). Para os demais setores, ao nível de significância de 5\% não é possível afirmar que há diferença significativa entre os coeficientes, mas as estimativas seguem o comportamento sugerido pelos autores no que se refere à influência dos retornos de escala sobre as estimativas de mark up.

Em termos de interpretação econômica dos resultados, de acordo com a teoria microeconômica, em setores com retornos crescentes de escala há uma tendência natural à concentração de mercado, já que maiores empresas se beneficiam desta característica e tendem a eliminar empresas que produzem em menor escala. Sob essa ótica, políticas públicas que visam à formação de grandes players nacionais com o intuito de expandir a participação brasileira no comércio internacional, apesar de serem prejudiciais sob a ótica da concorrência, podem fazer com que as empresas de setores com tais características produzam de maneira mais eficiente ao atuar em escala internacional. 
A relação positiva entre o mark up praticado e empresas exportadores encontrada por Loecker \& Warzynski (2009) evidencia esta relação.

Dessa forma, os setores veículos automotores e florestal, que apresentam retornos crescentes de escala de acordo com as estimativas da presente pesquisa, podem ter seus altos mark ups relacionados a esta característica. Contudo, para considerar a possibilidade de expansão de empresas nacionais no mercado internacional é necessária uma análise mais abrangente, já que outras considerações devem ser levadas em conta, como aspectos logísticos e concorrência internacional por exemplo.

\subsection{Análise de Robustez dos Resultados}

É relevante observar a relação entre preços de produtos e insumos praticados no mercado para obter evidências independentes das estimativas de mark up discutidas acima. Em indústrias nas quais uma única matéria prima é extremamente relevante no processo produtivo, a divisão do preço do produto final pelo preço desta matéria prima fornece uma aproximação plausível da relação entre preço e custo marginal, ${ }^{10}$ já que os custos de outros insumos são menos relevantes. Além disso, como o custo marginal não pode ser inferior ao preço de apenas um dos insumos, a divisão entre preço do produto e preço do insumo será maior do que a divisão do preço pelo custo marginal, o que tornaria esta relação de preços um limite superior para o mark up. Esta análise foi feita para as indústrias de siderurgia e de carnes bovinas e as relações de preços consideradas, além das estimativas obtidas na presente pesquisa, são apresentadas na tabela seguinte.

Tabela 4 - Resultados da pesquisa, resultados da literatura e relação de preços

\begin{tabular}{lcccc}
\hline & Siderurgia & Metalurgia básica & Carnes Bovinas & Alimentos \\
\hline Mark up Resíduo de Solow - indústrias & 4,45 & & 3,07 & \\
Mark up Resíduo de Solow - setores & & 2,86 & & 3,91 \\
Mark up Função de Produção - setores & & 2,96 & & 2,02 \\
Preço do produto & 656,67 & & 257,64 & \\
Preço do insumo & 131,13 & & 95,07 & \\
Relação de preço (Produto/insumo) & 5,01 & & 2,71 & \\
\hline
\end{tabular}

Fontes: Elaboração dos autores.

${ }^{10}$ Agradecemos a um parecerista anônimo a sugestão. 
O mercado de carnes bovinas é um candidato natural a este tipo de análise, uma vez que o boi gordo é insumo fundamental desta indústria. O conceito de boi teórico, com base no trabalho de Ledic, Tonhati e Fernandes (2000), permite estimar o preço final do boi considerando os cortes das carnes. Dessa forma, é possível harmonizar as informações em uma unidade comum de medida de preço final do boi para o consumidor e do custo de compra, que é o preço do boi gordo. De acordo com dados fornecidos por uma das maiores empresas do setor frigorífico sobre os preços de varejo da carne bovina no Estado de São Paulo, em condições de confidencialidade, bem como dados do CEPEA sobre o preço do boi gordo neste mesmo Estado, levando-se em conta as ponderações propostas para composição do boi gordo, a relação entre preço do boi teórico e preço do boi gordo para o período entre outubro de 2010 e setembro de 2012 é de 2,71.

Este resultado indica que os mark ups calculados por meio do resíduo de Solow para o setor alimentício e para a indústria de abatimento de carnes, respectivamente de 3,91 e 3,07, devem ser sobreestimados. Já o mark up de 2,02 estimado por meio da função de produção é factível com a evidência de preços considerada.

Outra indústria candidata a este tipo de análise é a siderúrgica. A produção de aço possui como insumo fundamental o minério de ferro, de forma que a divisão do preço do aço pelo preço do minério de ferro representa um limite superior para o mark up. $\mathrm{O}$ minério de ferro é uma commodity com preço formado em mercado mundial. Por isso, os dados fornecidos pelo Banco Mundial sobre o preço spot da tonelada do minério de ferro em dólares americanos é um bom indicativo de preço deste insumo. Já o aço possui diversos usos alternativos e, por isso, gera diferentes tipos de produtos. Foram considerados os preços da tonelada de lâminas quentes e de vergalhões também em dólares americanos. Os preços destes produtos são fornecidos pela MEPS e são referentes aos mercados dos países pertencentes aos BRIC (Brasil, Rússia, Índia e China). Considerando a média dos preços mensais entre outubro de 2011 e novembro de 2012, a relação entre o preço do minério de ferro e dos produtos de aço é de 5,01. A estimativa obtida por meio do resíduo de Solow para a indústria siderúrgica foi de 4,45 , o que está dentro do limite esperado. Para o setor de metalurgia básica o mark up estimado foi de 2,86 pelo resíduo de Solow e de 2,96 pela função de produção, 
valores próximos e em acordo com a hipótese de retornos constantes de escala para o setor, que não foi rejeitada no teste realizado. A diferença entre a estimativa calculada para a indústria e para os setores indica a grande heterogeneidade presente no setor metalurgia básica. O setor de metalúrgica básica apresenta mark up alto em relação aos demais, sendo que a indústria siderúrgica apresenta estimativa ainda maior, o que é evidenciado pela relação de preços analisada.

Levando em conta as estimativas de mark up por meio da função de produção, por estas serem robustas a diferentes retornos de escala, nove dos onze setores apresentaram preço mais do que duas vezes maior do que o custo marginal. Destaque para o setor florestal, que apresentou mark up de 3,81. Os setores de metalurgia básica, veículos automotores, equipamentos gerais e extrativistas seguem a lista das maiores relações entre preço e custo marginal, com estimativas de 2,95, 2,51, 2,43 e 2,42, respectivamente. No grupo das estimativas intermediárias estão os setores químico, têxtil, minerais não metálicos e alimentício, com mark ups de 2,38, 2,29, 2,19 e 2,02, respectivamente. Os setores com menor relação entre preço e custo marginal foram o de eletroeletrônicos, com mark up de 1,89, e o de máquinas e equipamentos, com estimativa de 1,60. A média dos mark ups obtidos por meio da função de produção estimados foi de 2,41 . No caso da relação entre preço e custo marginal obtida por meio do resíduo de Solow, esta média foi de 2,96. Ambas as estimativas apresentaram valores próximos à média da relação entre preço e custo marginal calculada por Hall (1988) para as indústrias norte-americanas entre 1953 e 1984, que foi de 2,79 para todos os setores e de 2,45 , desconsiderando os setores de transportes, comércio, serviços e finanças (setores que não foram analisados na presente pesquisa).

Dessa forma, os resultados são claros no que se refere à significativa diferença entre preço e custo marginal. Ao empregar uma metodologia robusta a diferentes retornos de escala e inédita para a indústria brasileira, os mark ups estimados são bastante superiores à unidade, mesmo considerando as diferenças entre as indústrias dentro de cada setor. Além da contribuição em termos de políticas públicas, tais resultados representam que deve haver problemas em estudos empíricos que partem da premissa de concorrência perfeita, uma vez que esta condição possui como uma de suas principais características a igualdade entre preço e custo marginal. 


\section{Conclusão}

Os resultados obtidos indicam que cuidados devem ser tomados em estudos empíricos que se baseiam na hipótese de concorrência perfeita na indústria brasileira. O preço e o custo marginal diferem para todos os setores analisados, com estimativas de mark up significativamente maiores do que a unidade. Dentre os onze setores analisados, nove apresentam preço mais do que duas vezes maior do que o custo marginal. Dessa forma, diversos trabalhos empíricos que assumem concorrência perfeita devem gerar resultados distorcidos. Conforme Hall (1986) evidencia e Ferreira e Guillén (2004) constatam empiricamente para o caso da indústria brasileira, desconsiderar os aspectos concorrenciais gera viés no cálculo da produtividade e do crescimento do produto. Os estudos que assumem concorrência perfeita não se limitam aos de crescimento econômico, sendo que modelos de equilíbrio geral computável, como o aplicado por Domingues et al. (2012), também devem apresentar importantes alterações em suas considerações caso os efeitos da diferença entre preço e custo marginal passem a ser considerados.

Além de ressaltar a necessidade de incorporar aspectos concorrenciais nos estudos empíricos, os resultados obtidos fornecem importantes indicadores para políticas de defesa da concorrência. As estimativas obtidas por meio da função de produção estimaram, em média, um preço 2,41 superior ao custo marginal para a indústria brasileira de extração e transformação. Apesar de este ser um resultado relevante, o mark up por si só não é suficiente para uma análise sobre o comportamento dos setores.

O resultado obtido é robusto a desvios da premissa de retornos constantes de escala. A esse respeito, no caso das indústrias florestais e de metalurgia básica os retornos crescentes de escala auxiliam a compreensão das altas estimativas, já que maiores empresas se beneficiam desta característica e tendem a se sobrepor às empresas que produzem em menor escala, o que naturalmente gera maior concentração no setor.

A busca pelos fatores determinantes das estimativas de mark up encontradas é uma possível extensão do esforço empreendido nesta pesquisa e deve aumentar e solidificar ainda mais as relevantes contribuições em termos de políticas públicas que a estimação da relação entre preço e custo marginal gera. 
Outra possível extensão do esforço empreendido nesta pesquisa é a análise dos resultados obtidos para estudos setoriais.

\section{Referências}

ANDERSON, T.W.; HSIAO, C. Estimation of dynamic models with error components. Journal of the American Statistical Association, American Statistical Association, v. 76, n. 375, p. 598-606, 1981. ISSN 01621459.

ARELLANO, M.; BOND, S. Some testes of specification for panel data: Monte carlo evidence and an application to employment equations. The Review of Economic Studies, Vol.58, No. 2, pp 277-297, 1991.

BLUNDELL, R.; BOND, S. GMM estimation with persistent panel data: an application to production functions. The institute for fiscal studies, Department of Economics, UCL, Working paper, 1998.

BURNSIDE, C.; EICHENBAUM, M.; REBELO, S. Labor hoarding and the business cycle. The Journal of Political Economy, v. 101, n. 2, p. 245-273, 1993.

CAMERON, A.; TRIVEDI, P. K. Microeconometrics: Methods and Applications. [S.1.: s.n.], 2005.

CEPEA - Centro de Estudos Avançados em Economia Aplicada. Disponível em: <http://www.cepea. esalq.usp.br/>

CHARLES, H.; PERLOFF, J. M. Can market power be estimated? Working Paper No 689,

Department of Agricultural and Resource Economics, UCB, UC Berkeley, 1993.

CLEZAR, R. V.; TRICHES, D.; MORAES, R. Poder de mercado, escala e a produtividade da indústria brasileira entre 1994 e 2007. In: Anpec Sul. [S.1.: s.n.], 2010.

DOMOWITZ, I.; HUBBARD, R. G.; PETERSEN, B. C. Business cycles and the relationship between concentration and price-cost margins. The RAND Journal of Economics, v. 17, p. 1-17, 1986.

FERREIRA, P. C.; GUILLÉN, O. T. de C. Estrutura competitiva, produtividade industrial e liberalização comercial no brasil. Revista Brasileira de Economia, v. 4, n. 58, p. 507-532, 2004.

GARCIA, F. A evolução da produtividade total de fatores na economia brasileira: uma análise do período pós-real. Núcleo de Pesquisas e Publicações, EAESP/FGV/NPP, 2003.

GRILICHES, Z.; MAIRESSE, J. Production Functions: The Search for Identification. [S.1.], 1995.

HALL, R. E. Market structure and macroeconomic fluctuations. Brookings Paper on Economic Activity, V. 17, p. 285-338, 1986.

HALL, R. E. The relation between price and marginal cost in U.S. industry. The Journal of Political Economy, v. 96, p. 921-947, 1988.

LEDIC, I.L., TONHATI, H., FERNANDES, L.O. Rendimento integral de bovinos após abate. Ciência Agrotécnica, v.24, n.1, p.272-277, 2000.

LOECKER, J. Recovering markups from production data. International Journal of Industrial Organization, v. 29, p. 350 - 355, May 2011.

LOECKER, J. WARZYNSKI, F. Markups and firm-level exporta status. NBER Working Paper series, 2009. Disponível em: <http://www.nber.org/papers/w15198>.

MEPS. Disponível em: <http://www.meps.co.uk/index.htm>

MUNDLAK, Y. Estimation of Production and Behavioral Functions from a Combination of Cross-Section and Time-Series Data. [S.1.]: Stanford University Press, 1963.

SHAPIRO, M. D. Measuring market power in U.S. industry. NBER Working Paper, n. 2212, 1987.

YCHARTS, World Bank. Disponível em: <http://ycharts.com/indicators/sources/world_bank>.

Estud. Econ., São Paulo, vol. 43, n.4, p. 687-710, out.-dez. 2013 


\section{ANEXO - Base de dados e estimativas complementares}
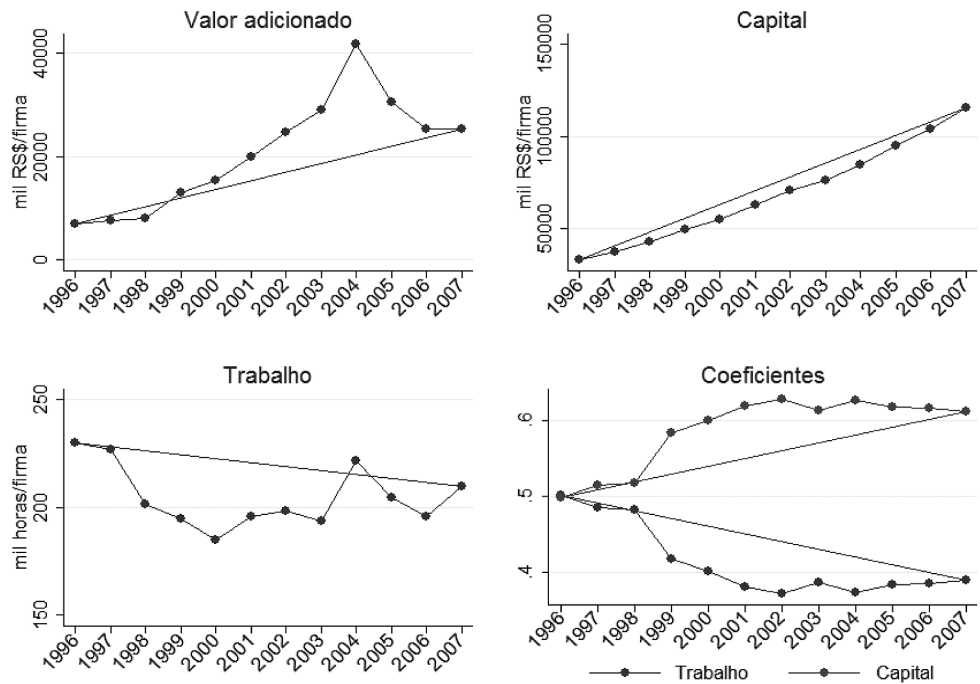

Figura 1 - Evolução do valor adicionado, capital, trabalho e coeficientes do trabalho e do capital.

Fonte: Elaborado pelos autores com dados da PIA-Empresa (IBGE).

Tabela 05 - Distribuição do valor agregado, do capital (em mil R\$), do trabalho (em mil horas) médias por setor e coeficientes de distribuição funcional da renda do capital e do trabalho

\begin{tabular}{lccccc}
\hline \multicolumn{1}{c}{ Setor } & Valor Adicionado & Trabalho & Capital & $\alpha_{i}^{n}$ & $\alpha_{i}^{k}$ \\
\hline Extrativista & $176.877,90$ & 335,72 & $83.558,10$ & 0,382 & 0,618 \\
Alimentos & $5.117,57$ & 99,06 & $26.327,95$ & 0,383 & 0,617 \\
Têxtil & $1.437,54$ & 55,22 & $5.701,67$ & 0,299 & 0,701 \\
Florestal & $9.723,54$ & 58,78 & $92.334,17$ & 0,497 & 0,503 \\
Química & $8.699,26$ & 61,52 & $38.367,98$ & 0,351 & 0,649 \\
Minerais não metálicos & $3.583,50$ & 37,34 & $41.637,30$ & 0,33 & 0,67 \\
Metalurgia básica & $7.361,69$ & 65,67 & $118.843,30$ & 0,397 & 0,603 \\
Máquinas e equipamentos & $4.942,83$ & 84,57 & $16.556,88$ & 0,422 & 0,578 \\
Eletroeletrônico & $5.179,94$ & 68,59 & $16.928,61$ & 0,481 & 0,519 \\
Equipamentos gerais & $2.167,74$ & 40,65 & $6.218,02$ & 0,441 & 0,559 \\
Veículos automotores & $27.224,03$ & 205,99 & $253.776,40$ & 0,441 & 0,559 \\
Outros & $20.662,24$ & 77,08 & $101.885,80$ & 0,48 & 0,52 \\
\hline
\end{tabular}

Fonte: Elaborado pelos autores com dados da PIA. 
Tabela 06 - Estimativas dos parâmetros da função de produção por GMM (com instrumentalização a partir da segunda defasagem) e por Pooled OLS

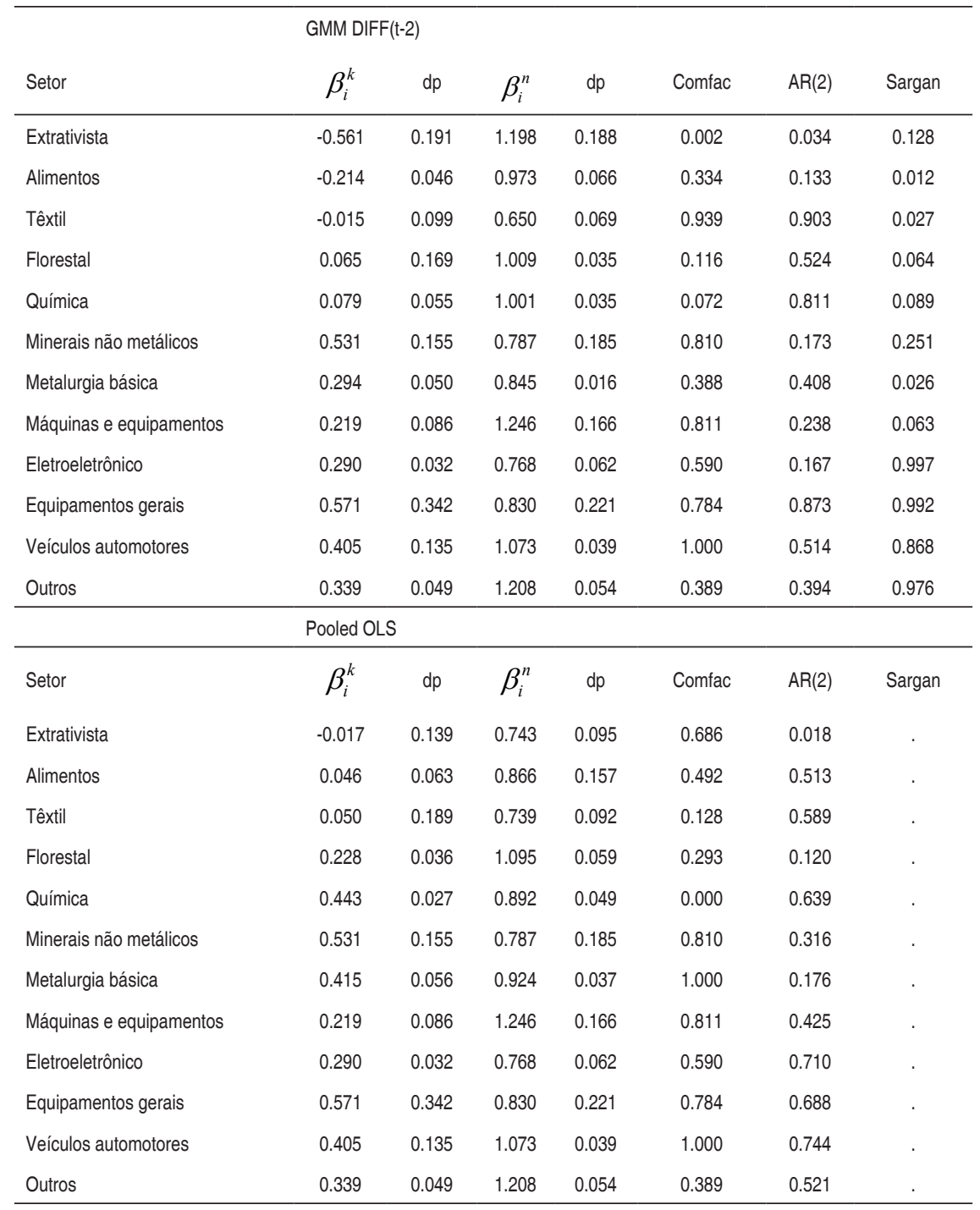

P-valor dos testes apresentados. Comfac: Ho = Restrições dos fatores comuns são válidas; Sargan: Ho = Instrumentalização para GMM adequada; AR(2): Não há correlação serial de segunda ordem do resíduo.

Fonte: Resultados da pesquisa. 
Tabela 07 - Estimativas de mark up em nível setorial por meio de resíduo de Solow (com taxa de câmbio como instrumento) - Cnae à esquerda e mark up à direita

\begin{tabular}{|c|c|c|c|c|c|c|c|c|c|c|c|}
\hline \multicolumn{2}{|c|}{ Têxtil } & \multicolumn{2}{|c|}{ Extrativista } & \multicolumn{2}{|c|}{ Eq. Gerais } & \multicolumn{2}{|c|}{ Alimentos } & \multicolumn{2}{|c|}{ Veículos Aut. } & \multicolumn{2}{|c|}{ El. Eletrônico } \\
\hline \multirow{2}{*}{171} & 2.761 & 100 & 2.397 & 331 & 2.239 & 151 & 3.071 & 341 & 2.568 & 301 & 4.656 \\
\hline & $(0.508)$ & & $(0.441)$ & & $(0.412)$ & & $(0.565)$ & & $(0.472)$ & & $(0.856)$ \\
\hline \multirow{2}{*}{172} & 2.063 & 112 & 1.608 & 332 & 2.042 & 152 & 3.752 & 342 & 2.496 & 302 & 3.109 \\
\hline & $(0.386)$ & & $(0.296)$ & & $(0.376)$ & & $(0.690)$ & & $(0.459)$ & & $(0.572)$ \\
\hline \multirow{2}{*}{173} & 2.444 & 131 & 7.562 & 333 & 2.160 & 153 & 7.405 & 343 & 1.925 & 311 & 2.050 \\
\hline & $(0.450)$ & & 1.391) & & $(0.397)$ & & $(1.362)$ & & $(0.354)$ & & $(0.377)$ \\
\hline \multirow{2}{*}{174} & 2.193 & 132 & 4.450 & 334 & 2.518 & 154 & 3.607 & 344 & 2.181 & 312 & 2.248 \\
\hline & $(0.403)$ & & $(0.818)$ & & $(0.463)$ & & $(0.663)$ & & $(0.401)$ & & $(0.413)$ \\
\hline \multirow{2}{*}{175} & 1.742 & 141 & 2.497 & 335 & 3.579 & 155 & 4.083 & 345 & 1.479 & 313 & 2.618 \\
\hline & $(0.320)$ & & $(0.459)$ & & $(0.658)$ & & $(0.751)$ & & $(0.272)$ & & $(0.481)$ \\
\hline \multirow{2}{*}{176} & 2.263 & 142 & 3.364 & \multicolumn{2}{|c|}{ M. não Met. } & 156 & 3.289 & 351 & 2.056 & 314 & 2.024 \\
\hline & $(0.416)$ & & $(0.619)$ & 261 & 2.772 & & $(0.605)$ & & $(0.378)$ & & $(0.372)$ \\
\hline \multirow{2}{*}{177} & 2.312 & & restal & & $(0.510)$ & 157 & 3.108 & 352 & 2.483 & 315 & 1.427 \\
\hline & $(0.425)$ & 201 & 2.537 & 262 & 6.800 & & $(0.572)$ & & $(0.457)$ & & $(0.263)$ \\
\hline \multirow{2}{*}{181} & 1.801 & & $(0.467)$ & & (1.251) & 158 & 2.567 & 353 & 3.210 & 316 & 2.014 \\
\hline & $(0.331)$ & 202 & 2.471 & 263 & 2.154 & & $(0.472)$ & & $(0.590)$ & & $(0.370)$ \\
\hline \multirow{2}{*}{182} & 2.044 & & $(0.455)$ & & $(0.396)$ & 159 & 4.304 & 359 & 3.130 & 319 & 2.450 \\
\hline & $(0.376)$ & 211 & 6.791 & 264 & 1.996 & & $(0.792)$ & & $(0.576)$ & & $(0.451)$ \\
\hline \multirow{2}{*}{191} & 2.599 & & (1.249) & & $(0.367)$ & \multicolumn{2}{|c|}{ Química } & \multicolumn{2}{|c|}{ Met. Básica } & 321 & 2.270 \\
\hline & $(0.478)$ & 212 & 3.433 & 269 & 2.546 & 241 & 4.155 & 271 & 4.611 & & $(0.417)$ \\
\hline \multirow{2}{*}{192} & 1.746 & & $(0.631)$ & & $(0.468)$ & & $(0.764)$ & & $(0.848)$ & 322 & 3.859 \\
\hline & $(0.321)$ & 213 & 2.904 & & tivista & 242 & 3.593 & 272 & 4.448 & & $(0.710)$ \\
\hline \multirow{19}{*}{193} & 2.066 & & $(0.534)$ & 100 & 2.397 & & $(0.661)$ & & $(0.818)$ & 323 & 3.463 \\
\hline & $(0.380)$ & 214 & 2.762 & & $(0.441)$ & 243 & 4.461 & 273 & 2.877 & & $(0.637)$ \\
\hline & & & $(0.508)$ & 112 & 1.608 & & $(0.820)$ & & $(0.529)$ & & e Eq. \\
\hline & & & & & $(0.296)$ & 244 & 2.825 & 274 & 3.895 & 291 & 2.149 \\
\hline & & & & 131 & 7.562 & & $(0.520)$ & & $(0.716)$ & & $(0.395)$ \\
\hline & & & & & (1.391) & 245 & 2.779 & 275 & 1.982 & 292 & 2.130 \\
\hline & & & & 132 & 4.450 & & $(0.511)$ & & $(0.365)$ & & $(0.392)$ \\
\hline & & & & & $(0.818)$ & 246 & 4.050 & 281 & 1.860 & 293 & 2.870 \\
\hline & & & & 141 & 2.497 & & $(0.745)$ & & $(0.342)$ & & $(0.528)$ \\
\hline & & & & & $(0.459)$ & 247 & 3.088 & 282 & 2.116 & 294 & 1.894 \\
\hline & & & & 142 & 3.364 & & $(0.568)$ & & (0.389) & & $(0.348)$ \\
\hline & & & & & $(0.619)$ & 248 & 2.702 & 283 & 2.168 & 295 & 2.718 \\
\hline & & & & & & & $(0.497)$ & & $(0.399)$ & & $(0.500)$ \\
\hline & & & & & & 249 & 2.828 & 284 & 2.214 & 296 & 1.863 \\
\hline & & & & & & & $(0.520)$ & & $(0.407)$ & & $(0.343)$ \\
\hline & & & & & & & & 289 & 2.379 & 297 & 1.857 \\
\hline & & & & & & & & & $(0.437)$ & & $(0.341)$ \\
\hline & & & & & & & & & & 298 & 2.712 \\
\hline & & & & & & & & & & & $(0.499)$ \\
\hline
\end{tabular}

Fonte: Resultados da pesquisa.

Desvio padrão entre parênteses.

Estud. Econ., São Paulo, vol. 43, n.4, p. 687-710, out.-dez. 2013 